요 Science and Engineering Research Council

A novel scenario for Neutrino Masses in Superstring-inspired GUT Models.

$$
\text { (1) }
$$


Science and Engineering Research Council

"The Science and Engineering Research Council does not accept any responsibility for loss or damage arising from the use of information contained in any of its reports or in any communication about its tests or investigations" 
RAL-92-035

\title{
A novel scenario for Neutrino Masses in Superstring-inspired GUT Models.
}

\author{
Stefano Ranfone and Elena Papageorgiu \\ Rutherford Appleton Laboratory \\ Chilton, OX11 OQX, England
}

\begin{abstract}
It is shown that the usual "super-suppression" of the standard neutrino masses in the context of the $S U(4) \otimes O(4)$ and the flipped $S U(5) \otimes U(1)$ models may be avoided in the presence of large VEV's of order $M_{G U T}$ for the right-handed sneutrinos, which have been introduced as a means to improve the mass relations between the down-type quarks and the charged leptons in the first of the two models.
\end{abstract}

June 1992 
In a previous paper [1], we had studied in detail the neutrino mass spectra of two supersymmetric GUT models, derived from Superstring Theory with Kac-Moody algebra level $\mathrm{K}=1$, and based on the "flipped" $S U(5) \otimes U(1)$ [2] and the $S U(4) \otimes O(4)$ [3] gauge groups. We recall that, starting from $S 0(10)$, these are the only two possible subgroups, which can be broken at the GUT scale $\left(M_{G} \simeq 10^{16} \mathrm{GeV}\right)$ down to the Standard Model $(\mathrm{SM})$ via incomplete $S 0(10)$ representations, and therefore do not require the presence of large self-conjugate and/or adjoint Higgs representations, in contrast to previous GUT models. This fact made them particularly attractive among other model candidates. Since predictions for neutrino masses and mixing are in fact one of the few testing grounds of these models (their effective low-energy behaviour is almost indistinguishable from other SUSY models), there has been recently some effort [4,1] in trying to understand and classify the different mass patterns that emerge, within the frame set by these models.

It was first thought $[2,3]$ that in both models all three neutrinos are essentially massless, having a super-suppressed mass of order $m_{\nu_{i}} \sim\left(m_{u_{i}} / M_{G}\right)^{2} M_{W} \leq 10^{-17} \mathrm{eV}$, where $m_{u_{i}}$ is the up-quark mass of the $i$-th generation, and $M_{W} \simeq 100 \mathrm{GeV}$ is the electroweak scale. It was then pointed out [4] that some of the neutrinos could get a mass of order $m_{u_{i}}^{2} / R \sim 10^{-4} \mathrm{eV}-100 \mathrm{keV}$, which would indeed have interesting phenomenological implications in astrophysics and cosmology, if large Majorana masses of order $R \sim 10^{8} \mathrm{GeV}$ could be generated radiatively at the two-loop level for the right-handed neutrinos $\nu^{c}$, via the so-called Witten mechanism [5]. This mechanism however becomes ineffective in all Superstring- derived models, because of the supersymmetric protection of the gauge hierarchy, which forbids the generation of radiative masses larger than a few TeV. Instead, in ref.[1] we have shown that the presence of suitable non-renormalizable terms, allowed, but not completely specified by the string models, can lead also to large masses for the right-handed neutrinos of the order $R \sim M_{G}^{2} / M_{S} \sim 10^{14} \mathrm{GeV}$, where $M_{S} \sim 10^{18} \mathrm{GeV}$ is the string unification scale. Some of the neutrinos would then have masses in the range of $10^{-10}-10^{-1} \mathrm{eV}$, and could play a role in resolving the solar neutrino problem à la MSW.

In this paper, motivated by the results obtained in Ref.[6], we would like to come back to the problem of the neutrino masses in the flipped $S U(5) \otimes U(1)$ and the $S U(4) \otimes O(4)$ models, showing that if the scalar partners of the right-handed neutrinos were to develop vacuum expectation values (VEV's) $\left\langle\nu^{c}\right\rangle \sim M_{G}$, this would open up the possibility of 
having also in the presence of SUSY neutrino masses in the $10^{-5}-10^{-3} \mathrm{eV}$ range without the need of non- renormalizable terms. Although the value of $\left\langle\nu^{c}\right\rangle$ was obtained in Ref.[6] from the study of the charged fermion spectra in the $S U(4) \otimes O(4)$ model, there is nothing preventing the right-handed sneutrinos from developing large VEV's also in the flipped $S U(5) \otimes U(1)$ model, so in principle one should consider both models. However, since the mass matrices have the same structure, we shall for simplicity discuss only the $S U(4) \otimes O(4)$ model and refer to Ref.[1] for the notation.

The gauge group of the model, which is isomorphic to the left- right symmetric group $S U(4) \otimes S U(2)_{L} \otimes S U(2)_{R}$, is spontaneously broken down to the SM via the VEV's of the neutral (scalar) components of $\mathbf{H}(\mathbf{4}, \mathbf{1}, \mathbf{2})$ and $\overline{\mathbf{H}}(\overline{\mathbf{4}}, \mathbf{1}, \mathbf{2}):\langle\mathbf{H}\rangle=\left\langle\bar{\nu}_{H}^{c}\right\rangle,\langle\overline{\mathbf{H}}\rangle=$ $\left.<\nu_{H}^{c}\right\rangle$. For simplicity we assume $\left\langle\nu_{H}^{c}\right\rangle=\left\langle\bar{\nu}_{H}^{c}\right\rangle \equiv M_{G}$, although their equality is implied by the minimization of the $F$ and the $D$ terms only in the flipped $S U(5) \otimes$ $U(1)$ model. This gives masses of order $M_{G}$ to the super-heavy gauge bosons and their corresponding superpartners, the gauginos, by means of the "absorption" of the would-be Goldstone (scalar) bosons, $u_{H}^{c}, \bar{u}_{H}^{c}, e_{H}^{c}, \bar{e}_{H}^{c}$, and a linear combination ${ }^{1}$ of $\nu_{H}^{c}$ and $\bar{\nu}_{H}^{c}$. As a concequence, all the fermionic components of $\mathbf{H}$ and $\overline{\mathbf{H}}$, which form massive states with the heavy gauginos, are also expected to have a mass of order $M_{G}$. In particular, without any loss of generality, we may assume for simplicity that the masses of the fermionic components of both $\nu_{H}^{c}$ and of $\bar{\nu}_{H}^{c}$ are equal to $M_{G}$. The mixing of these states with the other neutral states $\nu, \nu^{c}$ and $\phi$ is given by the various terms in the superpotential, shown in eq.(1) of Ref.[1], leading to the following mass-matrix for each generation of neutral leptons:

$$
\mathcal{M}_{\nu}^{0}=\left(\begin{array}{ccc}
0 & m_{u} & 0 \\
m_{u} & R & M_{G} \\
0 & M_{G} & X
\end{array}\right)
$$

Notice that there are two seesaw suppression mechanisms present in this simple mass matrix. One of them is due to the presence of massive singlet states $\phi$, which mix at the GUT scale with the right-handed neutrinos, leading to ultra-light neutrinos with a mass $m_{\nu} \sim\left(m_{u} / M_{G}\right)^{2} X$, where $X$, the mass scale of the $\phi$ 's, is comparable to the electroweak

1 While the corresponding orthogonal combination remains "uneaten", but still heavy and of order $M_{G}$. 
scale. The other seesaw mechanism is effective only when for some fermion generation the $\nu^{c}-\phi$ mixing is absent and it gives a neutrino mass proportional to $m_{u}^{2} / R$, as can also be seen by evaluating the diagram of Fig.2.

So, let us now discuss the effect of having a non-vanishing VEV for the right-handed sneutrino $\left\langle\nu^{c}\right\rangle$. The new terms, arising from the $\lambda_{2}$-term in the superpotential $\mathcal{W}_{4}$ given in eq.(1) of Ref.[1], are the mixing between $\phi$ and $\bar{\nu}_{H}^{c}$ and between $\nu^{c}$ and $\bar{\nu}_{H}^{c}$, which are proportional to $\left\langle\nu^{c}\right\rangle$ and $X_{2} \simeq M_{W}$, respectively. Merging these new terms with eq.(1), we get the complete $5 \times 5$ neutrino mass matrix in the basis $\left(\nu, \nu^{c}, \phi, \nu_{H}^{c}, \bar{\nu}_{H}^{c}\right)$ :

$$
\mathcal{M}_{\nu}=\left(\begin{array}{ccccc}
0 & m_{u} & 0 & 0 & 0 \\
m_{u} & R & M_{G} & 0 & X_{2} \\
0 & M_{G} & X & 0 & \left\langle\nu^{c}\right\rangle \\
0 & 0 & 0 & M_{G} & 0 \\
0 & X_{2} & \left\langle\nu^{c}\right\rangle & 0 & M_{G}
\end{array}\right)
$$

At a first glance one might wonder why in the limit $\left\langle\nu^{c}\right\rangle \rightarrow 0$ the $\nu_{H}^{c}$ and $\bar{\nu}_{H}^{c}$ mixing terms have not been considered in eq.(1) and in the previous works $[1,4]$. The point is that, when $\left\langle\nu^{c}\right\rangle$ goes to zero in eq.(2), the effective (Majorana) mass for the ordinary left-handed neutrino, as can also be seen by directly evaluating the diagram in Fig.(1), is given by:

$$
m_{\nu} \simeq\left(\begin{array}{llll}
m_{u} & 0 & 0 & 0
\end{array}\right)\left(\begin{array}{cccc}
R & M_{G} & 0 & X_{2} \\
M_{G} & X & 0 & 0 \\
0 & 0 & M_{G} & 0 \\
X_{2} & 0 & 0 & M_{G}
\end{array}\right)^{-1}\left(\begin{array}{c}
m_{u} \\
0 \\
0 \\
0
\end{array}\right) \simeq\left(\frac{m_{u}}{M_{G}}\right)^{2} X
$$

in agreement with the result obtained from eq.(1). Of course, in writing down the result in eq.(3) we have used the fact that in the supersymmetric case, and in the absence of nonrenormalizable terms, $R$ is comparable to the electroweak scale, as this is the case for the scales $X$ and $X_{2}$.

Now, in the presence of a large $\left\langle\nu^{c}\right\rangle$, the same procedure used above gives the following expression:

$$
m_{\nu} \simeq\left(\begin{array}{llll}
m_{u} & 0 & 0 & 0
\end{array}\right)\left(\begin{array}{cccc}
M_{R} & M_{G} & 0 & X_{2} \\
M_{G} & X & 0 & <\nu^{c}> \\
0 & 0 & M_{G} & 0 \\
X_{2} & \left\langle\nu^{c}>\right. & 0 & M_{G}
\end{array}\right)^{-1}\left(\begin{array}{c}
m_{u} \\
0 \\
0 \\
0
\end{array}\right) \simeq\left(\frac{\left\langle\nu^{c}>\right.}{M_{G}}\right)^{2} \frac{m_{u}^{2}}{M_{G}}
$$


This result, which again can be obtained directly from the diagram of Fig.3, shows that if $\left\langle\nu^{c}\right\rangle$ is comparable to $M_{G}$ for at least some of the three generations, one obtains neutrino masses which are not suppressed as in the case of $\left\langle\nu^{c}\right\rangle=0$, but rather correspond to the standard seesaw scenario where the right-handed scale has been replaced by the GUT scale.

The value of $\left\langle\nu^{c}\right\rangle \simeq M_{G}$ has not been chosen arbitrarily; it is the result obtained in Ref.[6] by improving the "naive" mass relations in the $S U(4) \times O(4)$ model between the down-type quarks and the charged leptons: $m_{d_{i}}=r m_{e_{i}}, r(\simeq 2.7)$ being the renormalization parameter introduced to compare quark- and charged lepton masses at a low mass scale $(\mu=1 \mathrm{GeV})$. As is well known, these relations are consistent with the actual masses only for the third generation. Although the problem associated with the masses of the first generation may be attributed to the dominance of non-perturbative QCD effects over the light $d$-quark mass, such an argument can hardly be used for the much heavier $s$-quark $\left(m_{s} \simeq \Lambda_{Q C D}\right)$. However, in Ref.[6] it was noticed that the presence of large VEV's for the right-handed sneutrinos can successfully modify some of the "naive" mass relations. In particular, keeping the successful equality $m_{b}=r m_{\tau}$ unchanged, while modifying the problematic one which relates $m_{s}$ to $m_{\mu}$, it turns out that only $\left\langle\nu_{\mu}^{c}\right\rangle$ has to be different than zero, leading to $[6]$ :

$$
m_{s}=\sqrt{z} r m_{\mu},
$$

where

$$
z=\left\{1+\left[\frac{<\nu_{\mu}^{c}>}{M_{G}}\left(\frac{r m_{\mu}}{v_{d}}\right)\right]^{2}\right\}^{-1},
$$

and $v_{d}=v \cos \beta \simeq v\left(m_{b} / m_{t}\right)$ is the VEV responsible for giving masses to the down-type quarks and charged leptons $(v=246 \mathrm{GeV})$. Using the actual value of the $s$-quark mass in eq.(5), one can then make a prediction for $\left\langle\nu_{\mu}^{c}\right\rangle$ :

$$
\frac{\left\langle\nu_{\mu}^{c}>\right.}{M_{G}} \simeq \frac{\lambda_{3} v}{m_{s}}\left(\frac{m_{b}}{m_{t}}\right) \sqrt{1-\left(\frac{m_{s}}{r m_{\mu}}\right)^{2}},
$$

in terms of the departure of $m_{s}$ from the naive mass relation. For a top quark mass of about $100 \mathrm{GeV}$ and for $\lambda_{3}=0.1$, which is a plausible choice, one obtains $\left\langle\nu_{\mu}^{c}\right\rangle \simeq M_{G}$, 
while $\left\langle\nu_{e}^{c}\right\rangle$ and $\left\langle\nu_{\mu}^{c}\right\rangle$ in this case are expected to be negligible compared to $M_{G}$. The impact of these results on the neutrino spectrum is that the electron and the tau neutrinos will still have a super-suppressed Majorana mass, as in eq.(3), while for the muon neutrino $m_{\nu_{\mu}} \simeq m_{c}^{2} / M_{G} \sim 10^{-5} \mathrm{eV}$, which, however, is still not large enough to have interesting phenomenological consequences. Nevertheless, since the $m_{d_{i}}=r m_{e_{i}}$ mass relations are absent in the flipped $S U(5) \otimes U(1)$ model, and there is nothing else preventing all three right-handed sneutrinos from developing VEV's at the GUT scale, one could still have a non-negligible tau neutrino mass $m_{\nu_{\tau}} \simeq m_{t}^{2} / M_{G} \sim 10^{-3} \mathrm{eV}$. In this case the solar neutrino deficit could be explained through the resonant oscillation of the much lighter $\nu_{e}$ 's into $\nu_{\tau}$ 's, refered to as the MSW mechanism.

\section{Figure Caption}

The diagrams which explain the origin of the neutrino masses in the different seesaw scenarios.

\section{References}

[1] E. Papageorgiu and S. Ranfone, Phys. Lett. B282 (1992) 89.

[2] I. Antoniadis, J. Ellis, J.S. Hagelin and D.V. Nanopoulos, Phys. Lett. 194B (1987) 231; 205B (1988) 459; 208B (1988) 209; 231B (1989) 65.

[3] I. Antoniadis and G.K. Leontaris, Phys. Lett. 216B (1989) 333; I. Antoniadis, G.K. Leontaris and J. Rizos, Phys. Lett. 245B (1990) 161; G.K. Leontaris, J. Rizos and K. Tamvakis, Phys. Lett. 243B (1990) 220; 251B (1990) 83.

[4] G.K. Leontaris and J.D. Vergados, Phys. Lett. 258B (1991) 111.

[5] E. Witten, Phys. Lett. 91B (1980) 81.

[6] S. Ranfone, RAL preprint, RAL-92-030 (to appear in Phys. Lett.B). 


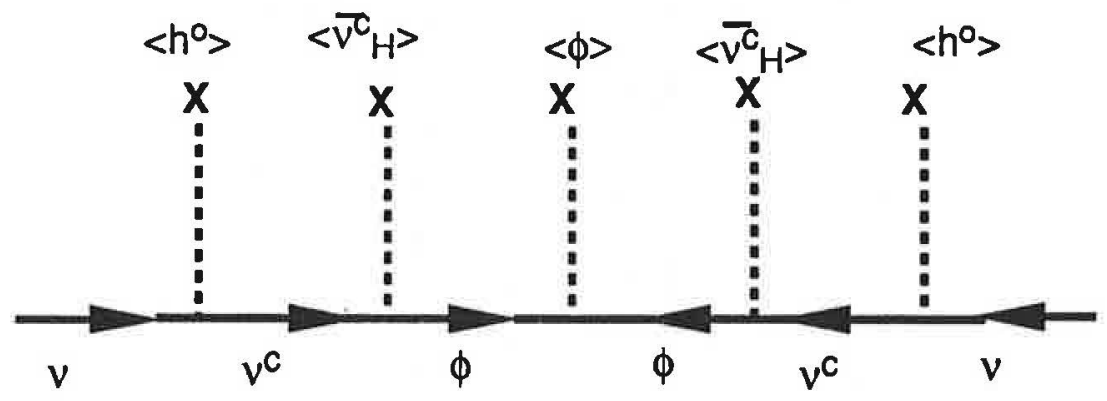

Fig. 1

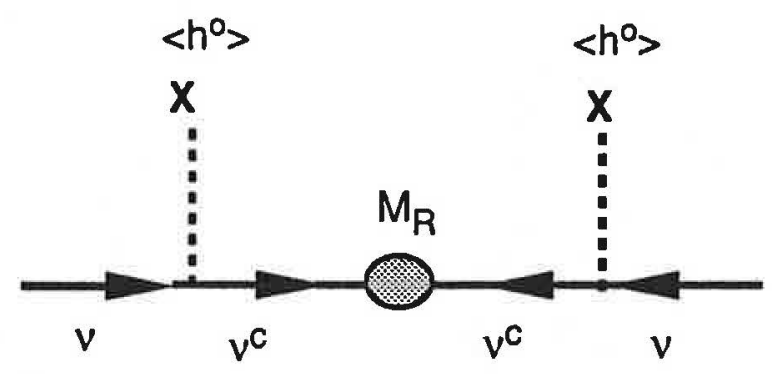

Fig. 2

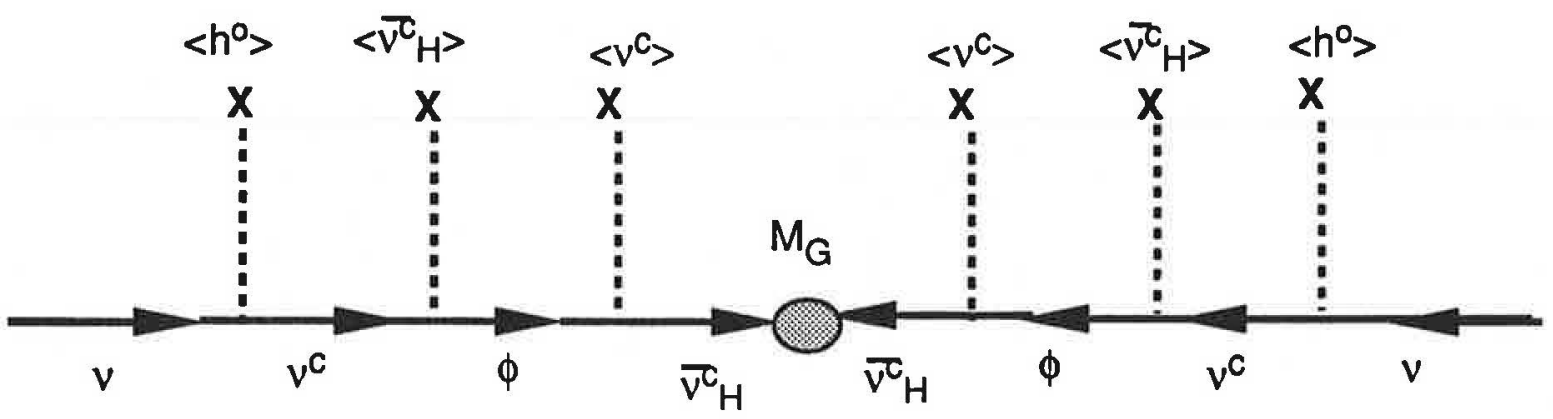

Fig. 3 


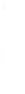



